\title{
The Influence of Self-talk on Children: An Analysis in Academic and Language Performance
}

\author{
Kexin Liu ${ }^{1, a, \dagger}$, Yujue Miao ${ }^{2, b, \dagger}$, Yuchen Yang ${ }^{3, c, \uparrow}$ \\ ${ }^{1}$ University of Bristol, Beacon house, Queens road, Bristol, BS8 1QU, England \\ ${ }^{2}$ Yunnan University of Finance and Economic, Kunming city, Yunnan, China \\ ${ }^{3}$ LinYi University, LinYi city, ShanDong Province, China \\ *Corresponding author.Email: ${ }^{a}$ Cecilia6661997@gmail.com, ${ }^{b}$ byncyzb@ynufe.edu.cn, ${ }^{c} 2673102199 @ q q . c o m$ \\ ${ }^{\dagger}$ These authors contributed equally.
}

\begin{abstract}
Children often construct their minds by observing and learning from their parents. Those messages from parents can change their beliefs and from their personalities by self-talks. These influences can often be demonstrated in the performances on tasks. In this study, it is hypothesis effected that different self-talk can have different effects on students' performance. According to the (Analysis of variance), the Influence of different types of self-talks is statistically and significantly different. As the persistence in the challenging academic task of positive group is higher than the other two groups (negative and neutral) in a pairwise contrast, positive self-talks can help the students do better. Finally, the overall correlations between different tasks (language and academic) are not found significant when applying correlations analysis. However, only the relation between vocabularies and academic performances of positive self-talks exists. Reasons for why positive self-talks are helpful in affecting psychological variables and helping enhance attention on performances are also discussed.
\end{abstract}

Keywords: self-talks, performance, effect, correlations

\section{INTRODUCTION}

It is known that self-talk is a statement of human language, and it plays a very important role in people's lives. For example, it can help improve cognition by analyzing problems. In addition, in dealing with psychological problems, people can relieve their pressure through positive self-narration to maintain a good mood. Vygotsky believes that is an important part of the development of higher psychological functions, all of which originates from the internalization of social interaction [1]. Most typically developing children start talking to themselves in the second or third year, around the time they start speaking in sentences. At first, selftalk is completely public and not always distinct from other direct speech. Its use continues into the fifth year, when a slow internalization process: self-talk is gradually cut off, high-pitched voices are harder to follow, and more and more children use the inner language. Public soliloquy, however, never completely disappears, and is still used throughout its life cycle according to Vicente and Martinez Manrique [2]. As Fahy mentioned [3], Luria and Vygotsky both put forward the importance of language as an intermediary tool used to solve problems and as a control characteristic of behavioral selfregulation. It is initially controlled and regulated by others in a child's growing environment. Over time, linguistic symbols develop while pass through interacting with the environment itself Children begin to use language to regulate themselves. The internalization of language is for planning and self-regulation.

Emerson proposed that language is the greatest tool for people to communicate with outside worlds [4]. It also a way to control behaviors [5]. And self-talk can improve performances and help people get new skills [6]. Many behaviors occur without conscious thinking, such as breathing. Others, however, rely on executive functions. Executive Functions (EFs) is a brain-based cognitive skill used to facilitate thinking and behavior. Studies showed that there is a strong correlation between language and EFs during early childhood [7]. Core EFs are working memory, inhibition (responses inhibition and interference control), and cognitive flexibility [8]. Working memory refers to temporary sticky notes in the brain. It's the ability to hold on new information so people can use it. Lots of learning processes can affect working memory, 
such as vocabulary acquisition [9]. Inhibition control describes the suppression of goal-irrelevant stimuli and behavioral responses [10]. Existing studies show that by the age of 7 , children have the conceptual understanding of when to inhibit responses, but they may not always translate them into efficient procedures [11]. During the transition from childhood to adolescence, the brain grows tremendously, causing a great change in cognitive capacities [12]. Moreover, evidence from the child in development suggests children's verbal ability is related to the their inhibition control [13]. Cognitive flexibility develops after the other two [14]. One aspect of cognitive flexibility is to change one's perspectives interpersonal, (e.g., "Let me say if I viewed if I can see this from your point of view."). When children use language to make speeches, it has a positive effect on cognitive capacities[15]. Based on existing studies, this paper propose that language learning has an important impact on executive functions. Moreover it is also expected that children can improve their language learning ability through positive self-talks.

Self-talks can be seen as a mental skill that can help interfere people's psychology [16]. It can affect not only people's behaviors but also performances in tasks from the inside spirits of their bodies. According to the content of self-talks, it can be either negative or positive. The genres of self-talks are very important as it forms different beliefs that have an influence on the actions of people. Hatzigeorgiadiset al. [17] found the selfconfidence will be increased, and cognitive anxiety will be declined by self-talks and affect academic performances. Later in his work, he discovered self-talks are closely related to the sports performance of athletes[17]. Negative self-talk often send pessimistic messages to their mind. Sentences such as 'You can't do it' or ' It is too difficult for you' will appear. The relationship of anxiety and negative self-talk is not expected to be positive, but state anxiety has a stronger relationship with thought-listing than think-aloud [18]. The performance of an athlete in a competition is negatively associated with the negative self-talks [17]. In contrast, the positive self-talks involve encouragements and praise (e.g., 'You are the best' or 'You can do it well').

This paper studies the relations of self-talks on children's persistence in academic performance. Instead of only focusing on the effect on academic performances, this paper further discovers the influence on language tasks and studies the relationships between language and academic tasks overall and within three groups (negative, positive, and neutral).

\section{METHOD}

\subsection{Participates}

In this study, data from 90 elementary school students between 6 and 7 years old are collected. Three different groups are divided based on self-talk (Positive self-talk, Negative self-talk, and Neutral self-talk) There are 30 people in each group. Besides, there is no significant difference in IQ, age, and gender of all participants.

\subsection{WISC-IV}

First, the WISC-IV (The Wechsler Intelligence Scale for Children) will be used to measure all students' intelligence. The WISC-IV is an individually administered intelligence test for children between the ages of 6 and 16. WISC-IV includes 10 core subtests and 5 additional subtests. There are four-index scores and one (Full Scare Intelligent Quotient) ranging from lowest (40) to highest (160) Test time is limited. Four-index scores contains: (1) The Verbal Comprehension Index (VCI), which includes the Vocabulary, Similarities and Comprehension; (2) The Perceptual Reasoning Index (PRI), which includes the Block Design, Picture Concepts and Matrix Reasoning subtest. (3) The Working Memory Index (WMI) includes the Digital Span and LatterNumber sequencing subtest; (4) The Processing Speed Index (PSI) includes the Coding and Symbol Search subtest.

\subsection{Self-talk Inventory}

Self-talk inventory (STI) is children's positive or negative natural self-talk. The most common self-talks responses are given in nine different hypothetical situations and used to the STI. In order to ensure validity, a situation involving social interaction is additionally added to the scale. In the SIT, children are asked whether they would say each of the 60 situations ( 3 positive and 3 negative for each of 10 situations) to themselves in response to the imaged situations using a Yes (3), Sometimes (2), No (1) response format.

\subsection{Language Measurement}

The Chinese version of Peabody picture vocabulary test is used to assess the receptive vocabulary of children. It contains 204 items. Each item has four pictures. After the administrator read the word, the children will ask to point out the picture that suits them best. The test will be carried out in 8 items at one time. If they make mistakes more than six times in one item, the test would be terminated. The final score is given by Subtract all errors and missing answers from the total number of questions (204). A higher score indicates a higher level of receptive language skills. 


\subsection{Academic task}

The questionnaires of mathematical part for grades 1 and 2 in the standard test of Wechsler Individual Achievement Test Second Edition[19] will be used to assess the performance that the three groups of self-talks can have on the academic performance of children. It tests children's ability of identifying and writing numbers and ability to solving problems by giving Numerical Operations and Math Reasoning with rising difficulties. We will let the children continue answering the questions even when they have consecutive errors. Before the test, the children will be informed to do as many as they can. The whole test must be completed within 30 minutes. Any answers given beyond the allotted time will not be scored. Only a pencil will be given during the test. The Grade-based standard scores given by the official WIATII information will be used to get the average number of correct questions in grade 1 and 2 . We will record the number of correct answers the students give after reaching the average number of correct questions. For instance, if the average number of right answers is 5, we will focus on counting the correct number of students from the 6th question. In this case, we can account for their ability to exceed the average. The exceed scores will show their persistence of how self-talk can encourage them to go further in the performance of an academic task. The consecutive errors or the correct numbers within the average number are not taken into consideration.

\section{PROCEDURE}

Participants will first take the self-talk inventory. Children will be grouped according to the performance of self-talk inventory. Subsequent language tests and academic tests will be conducted at the same session. Administrators of the two outcome measures will be blind to the group setting. There is a two-month interval between these two tests and the self-talk inventory.

\subsection{Data analytic plan}

The collection of data will be mainly from giving tasks to children. The whole team will read and comprehend all the information and collect and classify the data. The analytic method using in this study will be a one-way analysis of variance test (ANOVA test). The form of self-talk is considered to be the independent variable, and the performance in tasks is the dependent variable in this study. The null hypothesis has been posted in this study will be $\mathrm{HO}=$ all three types will not affect the performance of children. We recognize whether we should reject the null by figuring out the differences between groups of negative, positive, and neutral self-talks. If there is no difference between those groups, we will reject the null and fail to reject the null otherwise.
Furthermore, the correlations of data from language tasks and academic tasks will be examined. We will test the overall correlation of these two tasks implementing all data collected to see the relations between the performance of tasks. Then the correlations of two tasks within three different self-talk groups (positive, negative, and neutral) will be analyzed using data from each group.

\section{RESULT}

By applying the ANOVA test of overall data, we will reject the null, showing that the different types of selftalks will have different effects on the performances in tasks of children. The F-value indicates that the variance between the three groups is statistically and significantly different. We get a p-value with a value under 0.05 , showing the three groups are significantly different at a 5\% significant level. According to pairwise contrast, we compare the groups between negative vs. positive, negative vs. neutral, and positive vs. neutral. We find that the scores of positive self-talk academic tasks are higher than the other two groups, and the negative self-talks group has the lowest scores among them.

By applying the correlation analysis of overall data, no significant correlation will be found between language and academic performance in these three groups. However, we expect to find a positive correlation between vocabulary and performance in the positive self-talk group.

\section{DISCUSSION}

According to the result of the test, we find the positive self-talk group has the best persistence. The formation of this phenomenon has to do with the influence of parents. Parents educate their children about the consequences of their actions and create clear, consistent rules and contingencies for their children's actions. For example, verbal reminders not to eat before meals, can help children understand family rules and the consequences of eating before meals. Therefore, management language may also lead to better future performance in tasks requiring functional skills. Eventually, children may internalize their parents' management language into the self-talk they use to guide and plan their behavior, according to the research of Samantha W. Bindman, Annemarie H. Hindman, Ryan P. Bowles, and Frederick J. Morrisond[20] Self-talk can deepen students' understanding of classroom knowledge and logical thinking. As X.F.Liu[21] mentioned, children enrich and perfect the game using self-communication in words and realize the imitation and reproduction of people's behavioral norms in social life. In children's language development, they need to acquire enough vocabulary to make self-directed thinking plans. At the same time, they also need to acquire semantic relationships to compare features and thoughts. The above two things are inseparable from the help of logical reasoning provided 
by syntax according to the research of Fahy[3]. Bronowski proposed that the presence of language combined with emotional disengagement and behavioral inhibition makes it possible to reflect and plan, to test hypotheses, to think about the future, and to predict outcomes[22]. That may be the reason why they get higher scores.

The positive self-talk can help children focus more on tasks. Previous studies have shown the self-talk can affect psychological variables[23]. As Lopez and Snyder noted "internal conversions may help provide coherence and meaning to life experiences[24]." Self-talk is also used as a tool for elite football coaches to regulate pressure and maintain a proper focus during competitive situations[25]. Positive self-talk can change individual minds and actions. That, in turn, can have a significant impact on the face of any obstacles[26] And positive selftalk can help to enhance attentional focus and performance[17]. Moreover, positive self-talk can positively impact athletes' motivation[27], and the combination of a process goal, an analytic strategy[28]. Furthermore, as Hennecks said, positive self-talk focuses more on making the individual pay attention to specific tasks, which helps people perform better and reduce the influence of interfering factors in the plan. Moreover positive self-talk is a self-control technique that student makes better use of self-regulation[29]. Therefore, the positive self-talk can focus more on the specific plan without being distributed. It is also a challenge for selfregulation.

\section{CONCLUSION}

The study find that negative self-talk will be harmful to children on their academic achievements. In the forms of negative self-talk, children will gain inactive emotions from inside their body and mind as they tell themselves that they are likely to fail. The children with negative self-talks underperform in the schools, while those children keep encouraging themselves and make an effort inside will optimize their performance. The inactive influence may come from many ways. For instance, the children who are having self-talks often not having much confidence. They will hesitate on everything they do and deny their works even they have made the right decision. They might change their right answers into wrong, leading by the sense of diffident. The lack of confidence will influence their performances in academic tasks. Moreover, the kind of negative beliefs will make the children fear to do the hard questions at the beginning of the tasks and end up giving up without trying it.

This paper proposed hypothesis that the effects of children on their performances are from daily communications with their parents, however a questionnaire is not designed to investigate the real situation in the family, so there is no strong evidence to support the assumption that influence is from the family communications. Moreover, the assumption of improving the performance on tasks by increasing the concentration did not approve by a real investigation of data.The influence of parents on their children's language needs further research. Besides, self-talk is not interfered with within the group, so human intervention factors are not investigated.

\section{REFERENCES}

[1] L. Vygotsky, The genesis of higher mental functions. In The concept of activity in soviet psychology, ed. J.V. Wertsch, Armonk: Sharpe, 1981, pp. 144-188. Originally published in Russian in 1960.

[2] A. Vicente, F. Martinez Manrique, Inner speech: nature and functions, Philosophy Compass, vol. 6, 2011, pp 209-219.

[3] J. Fahy, Language and Executive Functions: Self-Talk for Self-Regulation, Perspectives on Language Learning and Education, 2014.

[4] R. W. Emerson, Essays \& lectures, Library of America, 1983.

[5] K. F. Jill, Language and Executive Functions: SelfTalk for Self-Regulation, 2018.

[6] E. Zetou, N. Vernadakis, E. Bebetsos, E. Makrakl, The Effects of Self-Talk in Learning the Volleyball Service Skill and Self-efficacy Improvement, 2012.

[7] D. Gooch, P. Thompson, C. Holme, The Development of Executive Functions and Language Skills in the Early School Years, 2016.

[8] B. Geurts, Making Sense of Self Talk, Review of Philosophy and Psychology, vol. 9, 2018, pp. 271285.

[9] Y. Guo, The Influence of Working Memory of Second Language Learning, 2016.

[10] J. Tiego, R. Testa, A. M. Bellgrove, C. Pantelis, S. Whittle, A Hierarchical Model of Inhibitory Control, 2018.

[11] M. S. Dowesett, J. D. Livesey, The Development of Inhibitory Control in Preschool Children: Effects of "Executive Skills", Developmental Psychobiology, vol. 36, issue 2, 2000, pp. 161-174.

[12] R. E. Baker, Q. Liu, R. Huang, A View from the Start: A Review of Inhibitory Control Training in Early Childhood, 2019

[13] C. K. Morasch, Bell Ann, M. The Role of Inhibitory Control in Behavioral and Physiological Expressions of Toddler Executive Function, Journal of Experimental Child Psychology, vol. 108, issue 3, pp. 593-606, 2011. 
[14] M. C. Davidson, D. Amso, L. C. Anderson, A. Diamond, Development of cognitive control and executive functions from 4 to 13 years: Evidence from manipulations of memory, inhibition, and task switching, Neuropsychologia, vol. 44, issue 11, 2006, pp. 2037-2078.

[15] F. L. Steger, Oxford Handbooks Online, 2009.

[16] J. B. Zimmerman, A. Kitsantas, Self-Regulated Learning of a Motoric Skill: The Role of Goal Setting and Self-Monitoring, 2008

[17] A. Hatzigeorgiadis, E. Galanis, Self-Talk Effectiveness and Attention, 2017.

[18] J. Lodge, G. Tripp, D. K. Harte, Think-aloud, thought-listing, and video-mediated recall procedures in the assessment of children's self-talk, Cognitive Therapy and Research, vol. 24, issue 4, 2000, pp. 399-418.

[19] D. Wechsler, Wechsler individual achievement testSecond UK edition, The Psychological Corporation, 2005.

[20] Samantha W. Bindman, Annemarie H. Hindman, Ryan P. Bowles, and Frederick J. Morrisond. The Contributions of Parental Management Language to Executive Function in Preschool Children, Early Child Res Q. Author manuscript; available in PMC 2014 Jul 1.

[21] X. F. Liu, The role of self-talk in the psychological development of children, Journal of Xinyang Normal University (Philosophy and Social Science Edition), issue 30, 2010, pp. 005.

[22] J. Bronowski, A Sense of the Future: Essays in Natural Philosophy, Cambridge, MA: The MIT Press, 1977.

[23] N. Walter, L. Nikoleizig, D. Alfermann, Effects of Self-Talk Training on Competitive Anxiety, SelfEfficacy, Volitional Skills, and Performance: An Intervention Study with Junior Sub-Elite Athletes, 2019.

[24] C. R. Snyder, S. J. Lopez, (Eds.). Handbook of positive psychology. Oxford university press, 2001.

[25] S. Freitas, C. Dias, A. Fonseca, Elite Soccer Coaches Use of Psychological Techniques, 2013.

[26] J. M. Cash, An Impact Study of the Safe Place Prayer Applied to Women Who Endured Childhood Sexual Abuse (Doctoral dissertation, Ashland University), 2020

[27] J. L. Van Raalte, B. W. Brewer, B. P. Lewis, D. E. Linder, et al., Cork! The effects of positive and negative self-talk on dart throwing performance,
Journal of Sport Behavior, vol. 18, issue 1, 1995, pp. 50-57.

[28] A. Kitsantas, B. J. Zimmerman, Self-regulation of motoric learning: A strategic cycle view, Journal of Applied Sport Psychology, vol. 10, issue 2, 1998, pp. 220-239.

[29] M. Hennecke, T. Czikmantori, V. Brandstatter, Doing Despite Dislike: Self-regulation in Everyday Aversive Activities, European Journal of Personality, Eur. J. Pers, 2018. 